\title{
Pulsar optical observation with the Very Large Telescope
}

\author{
A. Ray
}

Tata Institute of Fundamental Research, Bombay 400005, India

P. Lundqvist, J. Sollerman

Stockholm Observatory, SE-133 36 Saltsjöbaden, Sweden

B. Leibundgut

European Southern Observatory, Garching bei München, Germany

F. Sutaria

Inter Univ Centre for Astronomy \& Astrophysics, Pune 411007, India

\begin{abstract}
Optical data in the V-band gathered with the $8.2 \mathrm{~m}$ ESO Very Large Telescope (VLT) at the radio interferometric position of PSR 1706-44 are presented. The pulsar is close to a bright star in projection and was not detected. The pulsar magnitude limit must be fainter than $V=24.5$ for a distance of $\leq 2$. 0 from the bright star. In the outer gap model for an aligned rotor the optical flux should scale with the gammaray flux. For pulsars which emit pulsed gamma-rays but are not detected in the optical bands, the synchrotron cutoff frequency for the tertiary photons must be well below the optical frequencies and the magnetic and spin axes may be misaligned.
\end{abstract}

\section{Optical observation of PSR1706-44 \& pulsar radiation models}

Optical detection of pulsars aid in the development and constraining of theoretical models of pulsar electromagnetic radiation. PSR1706-44 belongs to the set of seven $\gamma$-ray pulsars detected by EGRET (Thompson et al. 1996). In the optical it has not been detected yet. Detection of the optical counterparts of radio and gamma-ray pulsars is often complicated by field crowding by other stars in the optical bands. The field of this pulsar was observed with the VLT-UT1 on August 19, 1998 (SV phase). The details of observation, analysis and theoretical implications are given in Lundqvist et al (1999). Chakrabarty and Kaspi (1998) (CK98) using the radio position of the pulsar as summarised in their paper estimate that the pulsar should lie 2.7 away from the star 1 . The combined error in position of the optical counterpart of the radio pulsar from various sources is $1^{\prime \prime} .0$. Measuring the background at a distance of $\sim 2$. 7 from nearby bright Star 1 in the VLT data shows that an artificial star with $V=25.5$ can be detected at more than $3 \sigma$ level. To estimate how bright a star one could hide in the PSF 

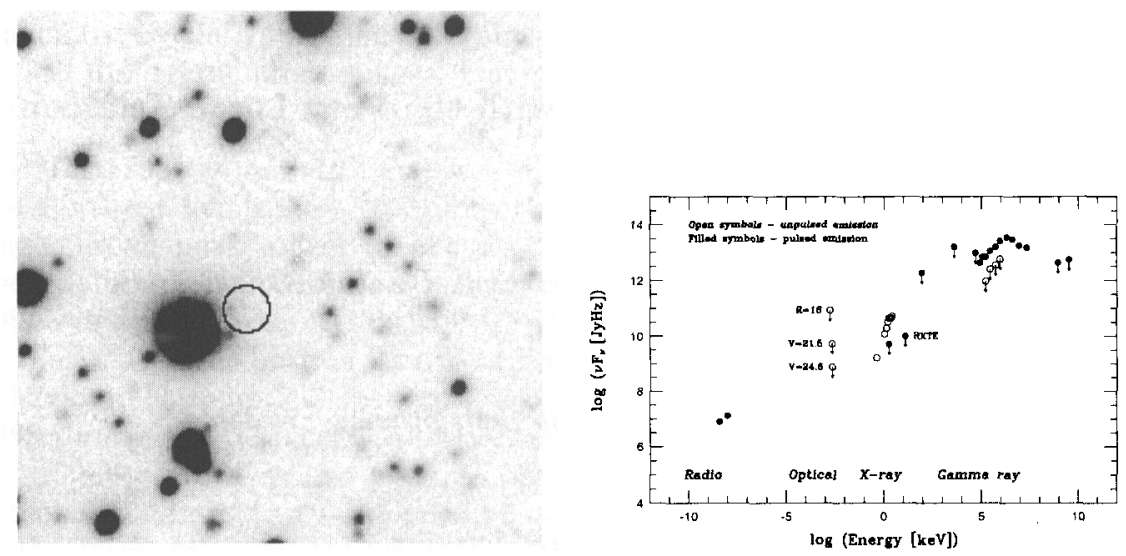

Figure 1. Left: The expected position of PSR1706-44 inside the $1^{\prime \prime} .0$ error circle centered 2.7 away from Star 1. Right: Multiwavelength spectrum from data compilations of Thompson et al. $(1996,1999)$. The points marked 'RXTE' and ' $R$ ' are from Ray et al (1999) and CK98 respectively. The VLT observation results marked $\mathrm{V}$ should bracket the dereddened upper limits: $V=24.6$ (minimum $A_{V}=0.9$ and the pulsar outside the PSF of Star 1) and $V=21.5$ assumes $A_{V}=3$.

of Star 1 we subtracted artificial stars from this position until a hole appeared in the background. It is possible to hide a point source $(V=25.0)$ at a distance of $\leq 2^{\prime \prime}$ from the Star 1. As an upper limit for a pulsar this close to Star 1 we claim $V=24.5$. The optical fluxes may be correlated with the gamma-ray photon fluxes in outer gap models (see e.g. Usov (1994) and Cheng, Ho \& Ruderman (1986a, 1986b)) for Vela-like pulsars. Assuming $F_{i}$ to be the same in both the $V$ and $R$ bands, the magnitudes predicted by the outer gap models are $R \lesssim 20.0$ and $V \lesssim 19.8$. Our faint $V$ limit, in comparison to the predictions of the standard outer-gap model, scaled from gamma-ray flux ( $V \lesssim 19.8$ ), requires a low frequency cutoff in its synchrotron emission spectrum. If the magnetic axis inclination with respect to spin axis $\chi \gtrsim \pi / 4$, the synchrotron cutoff frequency is $\sim 10^{13} \mathrm{~Hz}$ and in that case the flux of optical radiation may be very small.

\section{References}

Chakrabarty, D., Kaspi, V.M. 1998, ApJ, 498, L37 (CK98)

Cheng, K.S., Ho, C., Ruderman, M.A. 1986, ApJ, 300, 500

Lundqvist, P., Sollerman, J., Ray, A., Leibundgut, B. \& Sutaria, F., 1999, A \& A, 343, L15

Ray, A., Harding, A.K., Strickman, M.S. 1999, ApJ, 513, 919

Thompson, D.J. et al. 1996, ApJ, 385, 465

Thompson, D.J. et al. 1999, ApJ, in press (astro-ph/9811219)

Usov, V.V. 1994, ApJ 427, 394 